Research Article

\title{
Robust Geotechnical Design for Soil Slopes considering Uncertain Parameters
}

\author{
Xuejun Zhou $\mathbb{D}^{\mathrm{D}},{ }^{1}$ Wenxiong Huang, ${ }^{2}$ Jie Li $\mathbb{D}^{3},{ }^{3}$ and Ding Chen ${ }^{2}$ \\ ${ }^{1}$ College of Mathematics and Statistics, Huanggang Normal University, Huanggang, Hubei, China \\ ${ }^{2}$ College of Mechanics and Materials, Hohai University, Nanjing, China \\ ${ }^{3}$ School of Engineering, RMIT University, Melbourne, VIC, Australia \\ Correspondence should be addressed to Xuejun Zhou; zhouxj@hhu.edu.cn
}

Received 13 November 2019; Revised 2 February 2020; Accepted 22 February 2020; Published 16 March 2020

Academic Editor: Jan Vorel

Copyright $\odot 2020$ Xuejun Zhou et al. This is an open access article distributed under the Creative Commons Attribution License, which permits unrestricted use, distribution, and reproduction in any medium, provided the original work is properly cited.

Designing the geometry of soil slope is an effective treatment for preventing slope failure. How to deal with the uncertainties involved in soil parameters in geotechnical design is a main concern of geotechnical engineers. In this study, a robust geotechnical design for soil slopes (RGDS) approach was proposed, in which the Uncertainty Theory was introduced to describe explicitly the uncertainties involved in soil parameters. The uncertain reliability is often used to describe the risk of slope failure. The design robustness describing the insensitivity between the variation in the system response and the variation of input uncertain soil parameters was evaluated by the signal-to-noise ratio. The objectives of this design are to maximize the design robustness, minimize the excavation cost, and guarantee the safety (maximize the uncertain reliability). Therefore, the RGDS was formulated as a multiobjective optimization, and the optimal design can be determined based on the concepts of Pareto front and knee point. The proposed RGDS approach was illustrated through a numerical case of a two-layer slope design. The numerical results indicate that the RGDS approach is not only more intuitive and easier to follow but also more computationally efficient.

\section{Introduction}

Slope failure can trigger landslide and debris flow and cause significant damage to human life, buildings and infrastructure. Many precautions have been adopted to prevent slope failure. Among them, redesigning the geometry of a slope is a common treatment, which can change the mechanical balance condition of the slope to achieve the goal of prevention of slope failure.

Many uncertainties are generally encountered in slope stability analysis. The uncertainties in slope stability are mainly manifested in model uncertainty and parameter uncertainty [1]. The model uncertainty is caused by the difference between the actual behaviour of the slope system and its mechanical model. In fact, in slope stability analysis, compared with the parameter uncertainty, the uncertainty of the stability evaluation brought by the model uncertainty is much smaller [2]. Therefore, the uncertainty of soil parameters is the main factor for slope stability analysis.
Accordingly, how to deal with the uncertainties of soil parameters is a significant issue that the engineers and researchers should consider in analysing and designing slopes. It is often difficult to assign fixed values to the soil parameters with uncertainty. Appropriate methods should be developed to deal with such uncertainties.

Conventionally, the deterministic methods are used in geotechnical engineering designs. A conservative estimate of soil parameters was used to compute the safety factor that is required to be larger than an allowable safety factor derived from experiences. The uncertainties in soil parameters are not described explicitly in the process. The indeterministic methods allow for an explicit consideration of the uncertainties in soil parameters, and two main methodologies have been proposed in recent decades, i.e., probabilistic method and fuzzy method. Regarding the probabilistic method, Li and Lumb [3] discussed some improvements on the first-order second-moment (FOSM) probabilistic approach to slope design; Oka and $\mathrm{Wu}$ [4] studied the 
correlation between the failure probabilities of the different slip surfaces in the problems of slope stability; Christian et al. [5] described how probabilistic descriptions of soil parameters could be derived from field and laboratory data and applied in the analysis of slope stability; Baecher and Christian [6] gave a description of probabilistic methods that emphasizes both underpinnings of methodologies and their practical applications; Christian [7] proposed some issues, difficulties, and challenges about the application of reliability analysis and probabilistic method; Ang and Tang [8] applied the principles of probability and statistics to realistic problems related to engineering and the physical sciences, particularly civil and environmental engineering under uncertainty.

Generally, in the probabilistic method, input soil parameters are assumed to be random variables that have certain probability distributions on the basis of statistical characterization, and output failure probability can be computed to assess the stability of soil slopes. However, statistical characterization of input soil parameters usually originates from limited test data or local experiences, and it is often hard to guarantee the accuracy and so is the outcome of the probabilistic analysis. For the fuzzy method, Kacewicz [9] described soil parameters by fuzzy sets and considered the fuzzy method of slope stability estimation; Juang et al. [10] expressed uncertain soil parameters as fuzzy numbers on a set of intervals and handled soil parameter uncertainty in the stability analysis of slopes; Dodagoudar and Venkatachalam [11] presented the approach for the stability analysis of slopes incorporating fuzzy uncertainty; Giasi et al. [12] presented an algorithm, i.e., fuzzy first-order secondmoment (FFOSM), which used a fuzzy-based analysis applied to data processing in slope stability assessment; Park et al. [13] implemented the Monte Carlo simulation technique and uncertain reliability approach with fuzzy set theory in order to take into account the fuzzy uncertainties in the evaluation of the probability of failure; Gong et al. [14] presented a fuzzy set-based robust geotechnical design (RGD) approach for the design of earth slopes in which uncertain soil parameters are represented as fuzzy sets. In the fuzzy method, the statistical characterization of soil parameters is modelled with fuzzy sets (or fuzzy numbers), and then the outcome yields a fuzzy safety factor, the value of which would also be a fuzzy set (fuzzy number). The membership functions, such as triangular [15] and trapezoidal $[11,12]$, are used instead of the distributions in the probabilistic methods to describe the soil parameters with fuzziness. The membership functions in the fuzzy method eliminate one of the main drawbacks of the probabilistic method-the necessity for distribution functions in cases for we have scanty data. However, the construction of membership functions requires fuzzy experiments and subjective assumptions, and, thus, is also a difficult problem.

In fact, not all uncertainties involved in soil parameters could be considered random or fuzzy. For example, those uncertainties due to incomplete information because of cognitive sources cannot be handled satisfactorily in the probability or fuzzy theory, but can be treated appropriately in the Uncertainty Theory. The Uncertainty Theory was first proposed by Liu [16] for describing the imprecision of data. Compared with probability theory and fuzzy theory, the Uncertainty Theory has two advantages: one is that the Uncertainty Theory is more universal than probability theory and fuzzy theory and is suitable for dealing with uncertainty in the general sense. The calculation of the function distribution containing uncertain variables is much easier than that of random variables or fuzzy variables, which greatly reduces the computational complexity of evaluation indicators (such as safety factors) for slope stability. The Uncertainty Theory has been well developed [17] and widely applied [18-20]. Subsequently, Zhou et al. $[21,22]$ applied the Uncertainty Theory to estimate the stability of a slope. In their uncertain method [21], soil parameters are regarded as uncertain variables, and the distributions of these uncertain variables are built according to the structure of the soil layers of a slope. The safety factor is defined as the ratio of resisting and acting moments in an uncertain environment, and its expected value is therefore calculated by utilizing the operational laws of the uncertain variables. In addition, the concept of the uncertain reliability is also introduced in order to evaluate the risk of slope instability.

In the present study, a robust geotechnical design for soil slopes (RGDS) approach has been developed further based on the Uncertainty Theory. The robust geotechnical design is often a method of choice to deal with parameter uncertainties in the design, which is a methodology designed to achieve a certain level of design robustness, in addition to meeting the safety and cost requirements [23]. Coupled with soil slope design, a design is considered "robust" if the variation in the system response is insensitive to the variation of input uncertain soil parameters [24-26]. The objectives of this design are to maximize the design robustness and minimize the cost and guarantee safety (maximize the uncertain reliability). Therefore, the RGDS is a multiobjective optimization problem. The formulation and procedure of the RGDS approach could be applied on the basis of the robust geotechnical design reported in the literature [23].

In this paper, the deterministic stability analysis approaches for soil slopes employed in the subsequent study are reviewed, followed by stability analysis of soil slopes with the uncertain method and the formulation of the RGDS approach. Finally, a case of a two-layer slope is designed to verify the proposed RGDS approach.

\section{Deterministic Approaches for Slope Stability Analysis}

Several major deterministic methodologies have been developed to assess slope stability, i.e., limit analysis methods, limit equilibrium methods, numerical analysis methods (such as the finite element strength reduction approach), and so on. Among them, the limit equilibrium methods are the traditional slices methods, which were proposed by Fellenius et al. [27-30]. In our study, the Fellenius method is used. In fact, as is well known, the result of the Fellenius method is relatively smaller compared to those of other slices methods. 
However, the result of the Fellenius method has a certain safety reserve which is beneficial to the design. On the other side, an explicit formula of the safety factor in the Fellenius method is required in our uncertain analysis.

The determination of the critical slip surface is another important task during the process of using deterministic approaches. Generally, the critical slip surface is assumed to be a circular surface or a noncircular surface. For the convenience of research, the Fellenius method with a circular slip surface is employed for slope stability analysis. Of course, a noncircular slip surface can also be used.

2.1. The Fellenius Method. The Fellenius method assumes that the interslice forces are parallel to the base of each slice (see the forces $P_{i}$ and $P_{i+1}$ in Figure 1), which have a very minimal effect on the overall stability of the slope, so they can be neglected. Then, the safety factor $\left(F_{S}\right)$ can be defined as the ratio of the resisting moment divided by the overturning moment on the critical slip surface and can be directly obtained as follows [28]:

$$
F_{S}=\frac{\sum_{i=1}^{N}\left(N_{i}^{\prime} \tan \phi_{i}^{\prime}+c_{i}^{\prime} l_{i}\right)}{\sum_{i=1}^{N} W_{i} \sin \theta_{i}},
$$

where $W_{i}=\gamma_{i} v_{i}$ is the weight of the $i$ th slice in which $\gamma_{i}$ and $v_{i}$ denote the unit weight and the volume of the $i$ th slice, respectively; $N_{i}^{\prime}$ is the effective normal force of the $i$ th slice; $\theta_{i}$ is the inclination of the base of the $i$ th slice; $\phi_{i}^{\prime}$ is the effective friction angle of the $i$ th slice; $c_{i}^{\prime}$ is the effective cohesion of the $i$ th slice; $l_{i}$ is the width of the $i$ th slice basis.

2.2. Handling Pore Water Pressure. To design with hydrostatic pressure in slope stability analysis, it is desirable to determine the pore water pressure from a prescribed phreatic surface. However, this is usually not possible in the simplified methods (such as the Fellenius method). In order to include the effect of pore water pressure in the simplified methods, a soil parameter called pore water pressure ratio, $r_{u}$, is used instead [31]. The pore water pressure ratio can well model the pore water pressure that triggered failure, regardless of the preceding events or processes (e.g., rainfall intensity and duration, rate of infiltration) [32]. The pore pressure ratio is defined as the ratio between the total pore pressure and the total overburden pressure, or between the total upward force due to water pressure and the total downward force due to the water or overburden pressure [31].

If the failure surface is a circular slip surface as shown in Figure 2, the pore pressure ratio $r_{u}$ can be determined approximately by

$$
r_{u}=\frac{v_{\text {abea }}}{2 v_{\text {abcdea }}}
$$

where $v_{a b e a}$ and $v_{a b c d e a}$ denote the volumes of the soil masses abea and $a b c d e a$, respectively [31].

In the simplified methods of stability analysis, the pore pressure ratio is usually used to reduce the effective stress along the failure surface by a factor of $\left(1-r_{u}\right)$. Moreover,

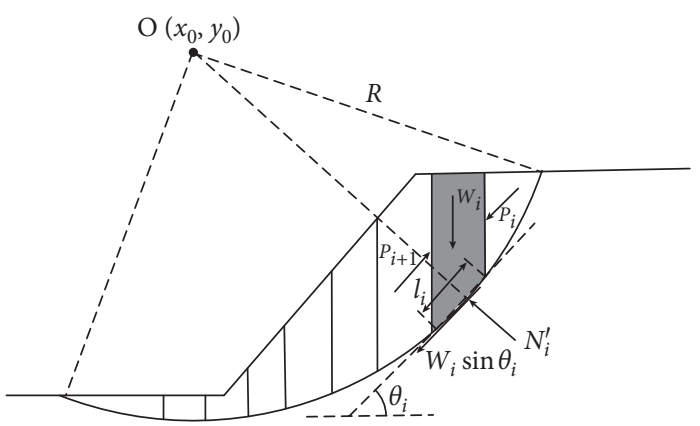

Figure 1: Fellenius method with a circular slip surface.

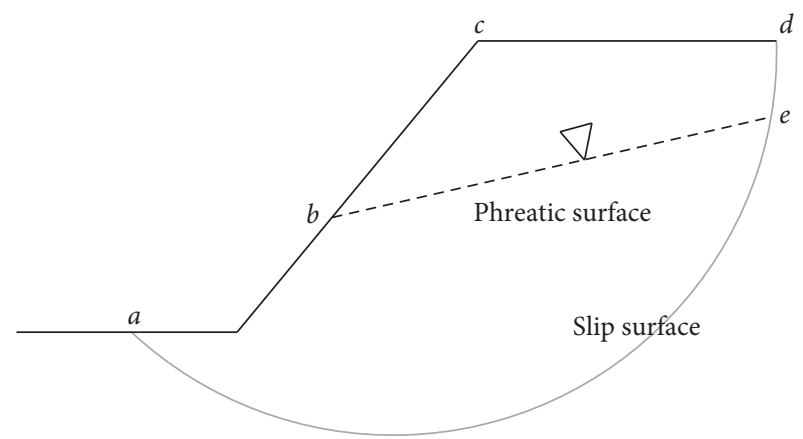

FIGURE 2: Determination of pore water pressure ratio.

Huang [31] suggested that the effective force $N_{i}^{\prime}$ in (1) can be replaced by

$$
N_{i}^{\prime}=\left(1-r_{u}\right) W_{i} \cos \theta_{i}
$$

This treatment can work well when the location of the failure surface must be known or estimated, so it is adopted in this study.

Although the pore pressure ratio is not an accurate representation of the pore pressure distribution along the slip surface, it is an index with an impact equivalent to that of the complex and unknown pore water pressure [32].

On the other hand, the obtained safety factor would change with the variation of the slip surface, and only the slip surface that yields the minimum safety factor can reflect the stability of the slope. Aiming to search for such a critical slip surface, a well-accepted commercial software for slope stability analysis, i.e., GeoStudio [33], was used to obtain the minimum safety factor based on (1), which can return the centre of this slip surface $\left(x_{0}, y_{0}\right)$ and its radius $R$ (see Figure 1).

\section{Analysis of Slope Stability Based on the Uncertainty Theory}

In the framework of the Uncertainty Theory, the soil parameters are regarded as uncertain variables. The stability of slopes may be more reasonably measured with the expected safety factor $\left(E\left[F_{s}\right]\right)$, rather than a deterministic $F_{s}$. Probabilistic or fuzzy methods are available to analyse the slope stability with uncertain soil parameters as inputs, where soil parameters are expressed as random variables or fuzzy sets. 
In the proposed study, the uncertainties associated with soil properties are described by the Uncertainty Theory [21].

3.1. Modelling Soil Parameters with Uncertain Variables. Let $\mathscr{L}$ be a $\sigma$-algebra over a nonempty set $\Gamma$, and an uncertain measure $\mathscr{M}$ is a set function satisfying normality, self-duality, and countable subadditivity. The triplet $(\Gamma, L, M)$ is an uncertainty space, and an uncertain variable is a function $\xi$ from the uncertainty space $(\Gamma, L, M)$ to the set of real numbers. The uncertainty distribution $\Phi$ of the uncertain variable $\xi$ is defined as $\Phi(x)=M\{\xi \leq x\}$ for any real number $x$, and its inverse function $\Phi^{-1}(\alpha)$ is the inverse uncertainty distribution of $\xi, \alpha \in[0,1]$ [17]. Soil parameters are usually determined through field tests, sampling, and laboratory tests. It is clear that uncertainty is involved in each of these steps. Therefore, the soil parameters in (1), namely, the unit weight $\gamma$, the effective friction angle $\phi^{\prime}$, the effective cohesion $c^{\prime}$, and the pore water pressure ratio $r_{u}$, are assumed to be uncertain variables in this study. Furthermore, they are assumed to be independent.

Note that there is often a certain correlation among soil parameters because of the influence of soil mineral composition, sedimentary conditions, stress history, and other factors. In our study, in order to facilitate the use of the theories of uncertain variables to conduct the analysis, we assume that these soil parameters are independent of each other.

3.2. Determining the Distribution Functions for the Soil Parameters. In the present research, the soil parameters $\gamma$, $\phi^{\prime}, c^{\prime}$, and $r_{u}$ are regarded as uncertain variables. Their uncertainty distributions are denoted by $\Phi_{1}(\gamma), \Phi_{2}\left(\phi^{\prime}\right)$, $\Phi_{3}\left(c^{\prime}\right)$, and $\Phi_{4}\left(r_{u}\right)$, respectively. They are determined on the basis of the structure of soil layers of the slope, where the highest conceivable value, lowest conceivable value, and most likely value (mean) are employed to build the distributions for different slopes. The following methods for building distributions have been proved effective in the literature [21].

For a homogeneous slope, if the mean values of soil parameters from laboratory tests are available, then such soil parameters are assumed to obey the linear distribution $L\left(a_{1}, a_{2}\right)$, where $a_{1}$ and $a_{2}$ are the lowest and highest conceivable values at a reasonable variation level, respectively (see Figure 3).

For a nonhomogeneous slope, if there is no soil stratigraphy between the soil layers $[34,35]$, that is, there are no different types of soil nested in each other, then the above method can also be used to build a corresponding linear

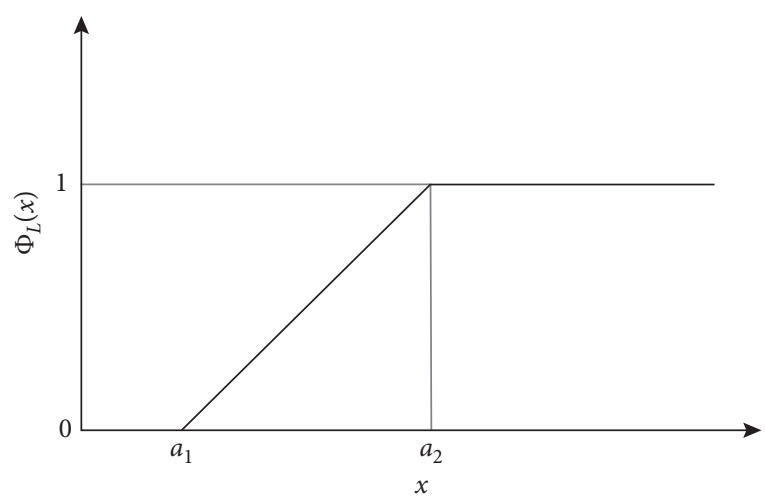

FIGURE 3: Linear uncertainty distribution.

uncertainty distribution for each parameter of each soil layer. But if there is soil stratigraphy between the soil layers, the following more direct method can be used. Normally, in the deterministic methods, the mean values of soil parameters of each soil layer are often available. Accordingly, these mean values can be used to build the distributions. The soil parameters may be assumed to obey the linear distribution $L\left(a_{1}, a_{2}\right)$ for a two-layer slope, where the smaller mean values of the two soil layers can be considered as the lowest conceivable values $a_{1}$, and the larger ones can be considered as the highest conceivable values $a_{2}$ on this occasion (see Figure 3); for a three-layer slope, the soil parameters can be assumed to obey the zigzag distribution $Z\left(a_{1}, a_{2}, a_{3}\right)$ (just like piecewise linear), where the minimum mean values of each parameter of the three layers can be considered as the lowest conceivable value of the corresponding parameter $a_{1}$, the maximum mean values can be considered as the highest conceivable value of the corresponding parameter $a_{3}$, and the middle ones can be considered as most likely value $a_{2}$ (see Figure 4).

Of course, the method may be developed to deal with more complicated slopes by introducing more memberships in Uncertainty Theory.

3.3. Evaluating Slope Stability with Safety Factor and Uncertain Reliability. When the soil parameters involved in (1) are expressed as uncertain variables, the safety factor becomes also an uncertain variable due to containing $\gamma, \phi^{\prime}, c^{\prime}$, and $r_{u}$. Therefore, the expected safety factor can be calculated via the inverse uncertainty distribution based on the operational laws of uncertain variable.

Firstly, the inverse uncertainty distribution of $F_{\mathrm{s}}$ can be expressed by

$$
\Psi^{-1}(\alpha)=\frac{\sum_{i=1}^{N}\left[\left(1-\Phi_{4}^{-1}(1-\alpha)\right) \Phi_{1}^{-1}(1-\alpha) v_{i} \cos \theta_{i} \tan \Phi_{2}^{-1}(\alpha)+\Phi_{3}^{-1}(\alpha) l_{i}\right]}{\sum_{i=1}^{N} \Phi_{1}^{-1}(1-\alpha) v_{i} \sin \theta_{i}}
$$

where $\Phi_{1}^{-1}(\alpha), \Phi_{2}^{-1}(\alpha), \Phi_{3}^{-1}(\alpha)$, and $\Phi_{4}^{-1}(\alpha)$ denote the inverse uncertainty distributions of $\gamma, \phi l, c l$, and $r_{u}$, respectively. It is noted that, because $F_{S}$ strictly increases with respect to $\phi_{\prime}$ and $c$ /but strictly decreases with $\gamma$ and $r_{u}$ 


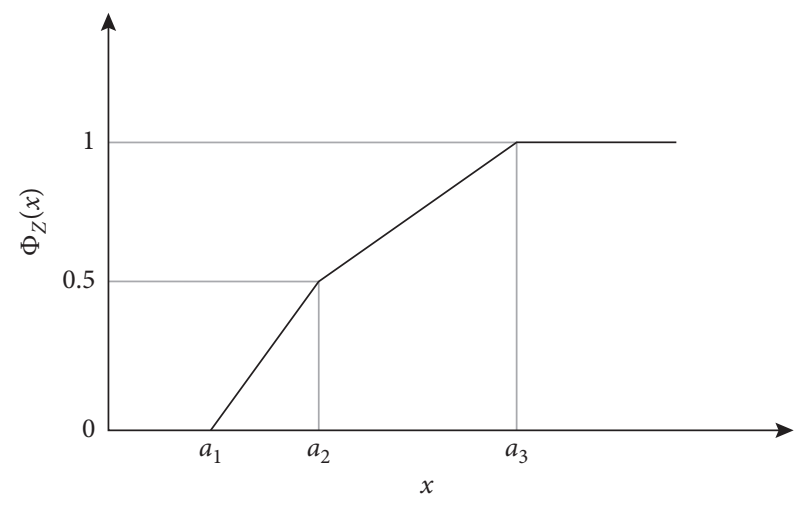

FIGURE 4: Zigzag uncertainty distribution.

from (1), the variable of the inverse distributions of $\phi \prime$ and $c$ । is $\alpha$, while that of $\gamma$ and $r_{u}$ is $1-\alpha$ in (4).

Through the integral of $\Psi^{-1}(\alpha)$, the expected value $E\left[F_{s}\right]$ and the variance $V\left[F_{s}\right]$ of the uncertain variable $F_{s}$ can be computed by

$$
\begin{aligned}
& E\left[F_{S}\right]=\int_{0}^{1} \Psi^{-1}(\alpha) \mathrm{d} \alpha, \\
& V\left[F_{S}\right]=\int_{0}^{1}\left(\Psi^{-1}(\alpha)-E\left[F_{S}\right]\right)^{2} \mathrm{~d} \alpha .
\end{aligned}
$$

The uncertain reliability is now introduced in the framework of Uncertainty Theory for further evaluation of the slope stability [21]. The uncertain reliability $\left(R_{e}\right)$ is defined as the uncertain measure that the safety factor is greater than one; i.e.,

$$
R_{e}=\mathscr{M}\left\{F_{S}>1\right\} \text {. }
$$

Actually, $R_{e}$ cannot be directly computed in our study, but Zhou et al. [21] have proven that $R_{e}$ is the root $\alpha$ of the equation

$$
\Psi^{-1}(1-\alpha)=1
$$

Therefore, substituting (4) into (8), $R_{e}$ can be obtained by solving (8). Note that sometimes (8) may not have a root. In this case, if $\Psi^{-1}(1-\alpha)>1$ for all $\alpha$, then we set the root $\alpha=1$, and if $\Psi^{-1}(1-\alpha)<1$ for all $\alpha$, then we set the root $\alpha=0$ [17].

\section{RGDS Method in Uncertain Environments}

4.1. Measure of Design Robustness. A design is considered robust if the variation in the system response is insensitive to the variation of input soil parameters. A measure of design robustness is therefore needed to estimate the response variation due to variation of the soil parameters. Juang et al. [23] adopted the failure probability as the design robustness in the probabilistic method. Gong et al. [14] proposed to measure the design robustness in the fuzzy-set-based method with the signal-to-noise ratio (SNR):

$$
\mathrm{SNR}=10 \lg \frac{E^{2}\left[F_{S}\right]}{V\left[F_{S}\right]} .
$$

In this work, SNR is adopted as a measure of design robustness in uncertain environments. In (9), $E\left[F_{s}\right]$ and $V$
$\left[F_{\mathrm{s}}\right]$ are the expected value and the variance of $F_{\mathrm{s}}$, respectively, and can be computed according to (5) and (6); "lg." represents the base-10 logarithm. Additionally, a larger SNR signifies smaller variability of the system response and, therefore, higher design robustness.

4.2. Optimization of the RGDS Method. As an example of the application of the proposed RGDS method, the design of a two-layer soil slope, as shown in Figure 5, is considered. The two-layer soil slope overlying a hard rock foundation needs to be manually excavated to meet the engineering requirement. ABCD is the original ground surface, and AFED is the designed ground surface. The goal of the design is to determine the angles of the cutting surface, i.e., $\alpha_{1}$ and $\alpha_{2}$. The RGDS method is used to find a candidate design which satisfies the demands of safety, design robustness, and cost simultaneously in a design domain (DD).

Robust design aims to make the product of a design insensitive to (or robust against) hard-to-control input uncertain soil parameters by adjusting easy-to-control design parameters. The essence of the proposed design approach is to consider robustness explicitly in the design process along with safety and cost requirements [23]. Therefore, this method is implemented through a multiobjective optimization, where all the design requirements such as safety, robustness, and cost are explicitly considered. In this optimization problem, the RGDS becomes a triobjective optimization where the objectives are to maximize the uncertain reliability $\left(R_{e}\right)$, maximize the design robustness (SNR), and minimize the cost $(C)$ synchronously. The mathematical formulation for the two-layer slope design is presented in Figure 6.

For the multiobjective optimization problem, as presented in Figure 6, the solution should provide insight into the trade-off relationships between safety, design robustness, and cost. However, actually, it is difficult to obtain a utopian solution that satisfies all conflicting objectives in DD simultaneously. Nevertheless, a set of nondominated optimal solutions may exist in DD, where each solution is characterized by the fact that an improvement of the design in any one objective can only be achieved at the cost of degradation in at least one other objective. This set of nondominated optimal solutions constitutes a Pareto front [36]. Figure 7 shows a conceptual illustration of a Pareto front in a biobjective space, with two objectives to be maximized. A possible optimization solution can be found in a biobjective space, where the Pareto front lies on the boundary of the feasible region [37]. On the Pareto front, none of the design points are superior or inferior to each other in all objectives [23]. For example, if $R_{e}$ and SNR are objectives 1 and 2 in Figure 7, respectively, the design $D_{1}$ is superior to the design $D_{2}$ with regard to SNR requirement, but is inferior to $D_{2}$ for $R_{e}$. Therefore, the optimal solutions on the Pareto front are the best compromise solutions that are optimal to all objectives. Generally, the Pareto front can be determined based on some optimization methodologies, in which the nondominated sorting genetic algorithm version II (NSGA-II) [38] is the most popular and successful one. In this study, 


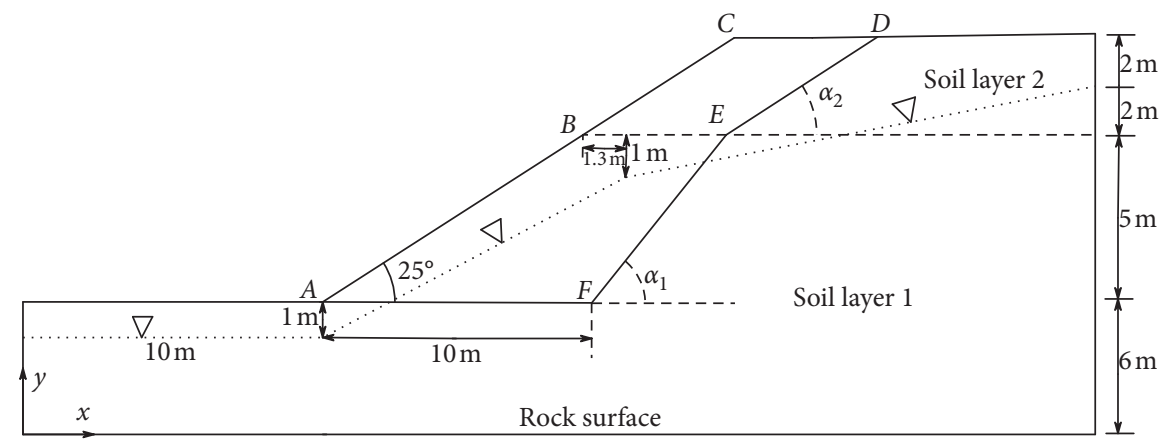

Figure 5: Cross section of a two-layer soil slope.

Find: Design parameters $\mathrm{d}=\left\{\alpha_{1}, \alpha_{2}\right\}$,

Subject to: $\mathrm{d} \in$ design domain (DD),

Objectives: Maximizing the uncertain reliability $\left(\mathrm{R}_{\mathrm{e}}\right)$, Maximizing the design robustness (SNR), Minimizing the excavation cost (C).

FIGURE 6: Formulation of multiobjective optimization for the design of a two-layer slope.

Objective 2

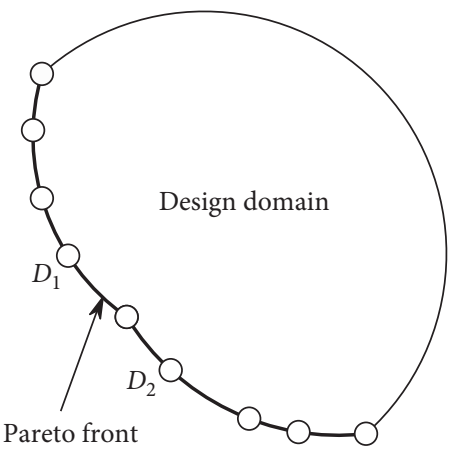

Objective 1

FIgUre 7: Conceptual illustration of a Pareto front in a biobjective space.

considering that DD in this optimization problem is discrete, an improved MATLAB code was written to seek the Pareto front.

Although the Pareto front provides a trade-off relationship among the three objectives that aid in informed decision-making, it is a laborious task to identify a single point on the Pareto front that yields the best compromise solution with respect to all the objectives. Actually, this point is the knee point which may lead to the most preferred design, since it needs a large deterioration in either objective to gain a small amount in the other objective [37]. Various approaches of selecting a knee point from the set of the Pareto front have been proposed, such as the compromise programming approach [39], the marginal rate of substitution approach [40], the pseudoweight vector approach [41], and the evolutionary multiobjective optimization (EMO) approach [42]. In this paper, the utility-based EMO procedure is applied to obtain the knee point on the Pareto front.

In this method, a linear utility function should firstly be given as

$$
U(\mathbf{d}, \lambda)=\sum_{i=1}^{3} \lambda_{i} f_{i}(\mathbf{d}),
$$

where $f_{i}(\mathbf{d})$ are objective functions to be minimized, i.e., $f_{1}(\mathbf{d})=-\mathrm{RI}, f_{2}(\mathbf{d})=-\mathrm{SNR}$, and $f_{3}(\mathbf{d})=\mathrm{C} ; \lambda=\left\{\lambda_{1}, \lambda_{2}\right.$, $\left.\lambda_{3}\right\}$, and the sum of all the $\lambda_{i} \in[0,1]$ equals 1 ; all $\lambda_{i}$ are equally likely. Branke et al. [42] suggested that the sampling of $\lambda_{i}$ can be done either randomly or in a systematic manner. In our study, different random values of $\lambda_{i}$ are generated with an assumption of uniform distribution.

Then, a marginal utility function can be written as

$$
\bar{U}\left(d_{n}, \lambda\right)=\min _{m \neq n}\left[U\left(d_{m}, \lambda\right)-U\left(d_{n}, \lambda\right)\right],
$$

where $d_{m}$ and $d_{n}$ are the candidate design points on the Pareto front. Accordingly, the expected marginal utilities can be approximated simply by sampling for each nondominated optimal design on the Pareto front, i.e., by calculating the marginal utility for all nondominated optimal designs for a number of randomly chosen utility functions, and taking the average as expected marginal utility [42]. The design which leads to the maximum expected marginal utility is exactly the knee point on the Pareto front.

\section{RGDS of Soil Slope: Case Study}

In this section, the proposed RGDS method will be applied to designing a case of the two-layer soil slope as shown in Figure 5 .

5.1. Brief Summary of the Case of Soil Slope. As shown in Figure 5, the total height of the soil slope is $15 \mathrm{~m}$, and the slope is composed of two layers of soil. The height of the lower layer (soil layer 1 ) is $11 \mathrm{~m}$, and the mean values of unit weight, effective angle of internal friction, and effective cohesion are taken as $20.0 \mathrm{kN} / \mathrm{m}^{3}, 23.0^{\circ}$, and $11.0 \mathrm{kN} / \mathrm{m}^{2}$, respectively. The height of the upper layer (soil layer 2) is 
$4 \mathrm{~m}$, and the mean values of unit weight, effective angle of internal friction, and effective cohesion are taken as $18.2 \mathrm{kN} /$ $\mathrm{m}^{3}, 18.0^{\circ}$, and $9.0 \mathrm{kN} / \mathrm{m}^{2}$, respectively. The upstream waterhead is $13 \mathrm{~m}$, and the downstream waterhead is $5 \mathrm{~m}$. In order to facilitate the treatment of the effect of hydrostatic pressure on stability of the slope, the condition of pore water pressure is represented by the pressure line in Figure 5. The angle $\angle B A F$ equals $25^{\circ}$. When assessing the stability of the soil slope, the engineers found that there might be a risk of instability, and it was recommended to carry out reinforcement. Due to engineering and other reasons, there must be a certain amount of space at the corners; that is, the length of $\mathrm{AF}$ is fixed at $10 \mathrm{~m}$.

The unit weights $\gamma_{1}, \gamma_{2}$, the effective friction angles $\phi_{1}^{\prime}, \phi_{2}^{\prime}$, the effective cohesion $c_{1}^{\prime}, c_{2}^{\prime}$, and the pore water pressure ratios $r_{u 1}, r_{u 2}$ of two soil layers are treated as uncertain variables. Assume that there is soil stratigraphy between the two soil layers in this case.

The pore water pressure ratios can be estimated with (2), i.e., $r_{u 1}=0.27, r_{u 2}=0.07$, whereas the volumes can be computed after determining the slip surface for the original slope. At this time, the centre of the slip surface is (15.33, 25.22), and the radius is $19.95 \mathrm{~m}$ (the number of slices was set to 30 in GeoStudio). In fact, when the number of slices is set to 30,60 , and 90 in GeoStudio, respectively, the centres of the slip surface are all around $(15.33,25.22)$ and the radii are all about $19.95 \mathrm{~m}$. This indicates that the numerical results are not sensitive to the number of slices. Therefore, in order to simplify the calculation, setting the number of slices to 30 is enough in our study.

The most likely values (or the mean) of the other uncertain variables were assumed to have been estimated based on limited test data with engineering judgment [14], as provided in Table 1 . These values were used to build the uncertainty distributions for each uncertain soil parameter.

As mentioned in Section 3.2, the soil parameters in this case can be assumed to obey the linear distribution, and their inverse uncertainty distributions can be written as

$$
\begin{gathered}
\Phi_{1}^{-1}(\alpha)=18.2(1-\alpha)+20.0 \alpha \\
\Phi_{2}^{-1}(\alpha)=\frac{18.0}{180} \pi(1-\alpha)+\frac{23.0}{180} \pi \alpha \\
\Phi_{3}^{-1}(\alpha)=9.0(1-\alpha)+11.0 \alpha \\
\Phi_{4}^{-1}(\alpha)=0.07(1-\alpha)+0.27 \alpha
\end{gathered}
$$

TABle 1: Assumed soil parameters of the two-layer slope.

\begin{tabular}{lcc}
\hline Soil parameter & Soil layer 1 & Soil layer 2 \\
\hline Unit weight $\left(\mathrm{kN} / \mathrm{m}^{3}\right)$ & 20.0 & 18.2 \\
Angle of internal friction $(\circ)$ & 23.0 & 18.0 \\
Cohesion $\left(\mathrm{kN} / \mathrm{m}^{2}\right)$ & 11.0 & 9.0 \\
Pore water pressure ratio & 0.27 & 0.07 \\
\hline
\end{tabular}

5.2. Estimation of Cost and Determination of Design Domain. In order to design the geometry parameters $\alpha_{1}$ and $\alpha_{2}$, the cost $\mathrm{C}$ and the design domain (DD) in Figure 6 should be determined. The excavation cost $\mathrm{C}$ can be expressed simply by the excavation volume (i.e., the area of polygon $A B C$ $D E F A$ in Figure 5) per longitudinal length $\left(\mathrm{m}^{3} / \mathrm{m}\right)[14,43]$ :

$$
C=90+\frac{32.5}{\tan \alpha_{1}}+\frac{8}{\tan \alpha_{2}}-\frac{40.5}{\tan 25^{\circ}}
$$

It is worth mentioning that other more complicated models for estimating the cost can also be used.

In the process of slope construction, in order to ensure the feasibility and operability, the design parameters $\alpha_{1}$ and $\alpha_{2}$ should be taken values from DD. A discrete DD is obtained from a preliminary assessment with engineering judgment:

$$
\mathrm{DD}=\left\{\left(\alpha_{1}, \alpha_{2}\right) \mid \alpha_{1}, \alpha_{2} \in\left\{25^{\circ}, 26^{\circ}, 27^{\circ}, \ldots, 50^{\circ}\right\}\right\} .
$$

Of course, different design domains may be considered, and the results would be similar. If the optimization of the RGDS of the soil slope is limited to the discrete domain as in (15), then DD includes $26 \times 26=676$ candidate designs. Specifically, the goal of design is to find out the most-preferred excavation angles $\alpha_{1}$ and $\alpha_{2}$ from the 676 candidate designs.

5.3. Calculation of Uncertain Reliability and Design Robustness. For different candidate designs in DD, the slip surface that yields the minimum safety factor can be determined by the software GeoStudio, in which the number of slices is set to 30 for all the 676 candidate designs.

The required values of variables $v_{i}, \theta_{i}$, and $l_{i}$ in (4) can be returned by GeoStudio. Substituting (12) and (13) into (4), and combining (8), $R_{e}$ is equal to the $\operatorname{root} \alpha$ :

$$
\frac{(0.93-0.20 \alpha)(1.8 \alpha+18.2) \tan (0.13-0.03 \alpha) \pi \sum_{i=1}^{30} v_{i} \cos \theta_{i}+(11-2 \alpha) \sum_{i=1}^{30} l_{i}}{(1.8 \alpha+18.2) \sum_{i=1}^{30} v_{i} \sin \theta_{i}}-1=0 .
$$

In order to calculate the SNR via (9), $E\left[F_{s}\right]$ can be firstly obtained by substituting (4) into (5); then, $V\left[F_{\mathrm{s}}\right]$ can also be obtained by substituting (4) and (5) into (6).
5.4. Relationships Between the Uncertain Reliability, the Design Robustness, and the Cost. For the triobjective optimization shown in Figure 6, it becomes a biobjective 
optimization without considering one of the objectives. As thus, the relationships between any two of the uncertain reliability $\left(R_{e}\right)$, the design robustness (SNR), and the cost $(C)$ in $\mathrm{DD}$ can be studied in this subsection.

Figure 8 shows the relationship between the uncertain reliability $\left(R_{e}\right)$ and the cost $(C)$ by plotting the values for all 676 candidate designs. It is noted that Figure 8 is just to provide a baseline reference without consideration of the design robustness. Figure 8 demonstrates that a trade-off relationship exists between the uncertain reliability and the cost in the design of soil slopes; i.e., the uncertain reliability tends to increase with the increase of the cost. More remarkably, the boundary of all discrete design points, as shown in Figure 8, actually establishes a Pareto front, and the Pareto front is a set of nondominated optimal designs in relation to maximizing the uncertain reliability and minimizing the cost. In all the 676 discrete design points, 64 design points are obtained as the nondominated optimal designs that belong to the Pareto front. On the other hand, the knee point is also shown in Figure 8 to find the best-compromise design from a Pareto front. The knee point is identified in Figure 6 using the marginal utility function-based procedure as described in (10) and (11). It can be found that a slight increase in cost can sharply increase the uncertain reliability on the left side of the knee point, but on the right side of the knee point a slight increase in the uncertain reliability can lead to a remarkable increase in the cost. As a result, the knee point $\left(\alpha_{1}=42^{\circ}, \alpha_{2}=39^{\circ}\right)$ can be taken as the most preferred between $R_{e}$ and $C$.

Figure 9 shows the relationship between the design robustness and the cost in DD. This trade-off relationship is observed with the Pareto front that is determined by 47 nondominated optimal designs where the uncertain reliability is not inspected. Moreover, the knee point $\left(\alpha_{1}=42^{\circ}\right.$, $\alpha_{2}=49^{\circ}$ ), which yields the best-compromise design between SNR and C, is obtained.

Finally, the relationship between the uncertain reliability and the design robustness in DD is shown in Figure 10. It can be seen that the design robustness almost increases with the uncertain reliability. Actually, when the suggested approach is applied to determine the Pareto front in this case, there are only two nondominated optimal designs on the Pareto front (see Table 2). As a result, it is not necessary to determine the Pareto front and find the knee point in this case, and the result indicates that a higher safety level (uncertain reliability) is achieved by a more robust design.

More detailed results for Figures $8-10$ are given in Table 2 .

5.5. RGDS of the Soil Slope. In Figure 11, all the 676 discrete candidate designs are presented in the three-dimensional space of $\left(R_{e}, \mathrm{SNR}\right.$, and C). The Pareto front which consists of 84 nondominated optimal design points is marked by circles in red. The knee point among the Pareto front is marked by a solid red circle which corresponds to the design parameters $\alpha_{1}=47^{\circ}$ and $\alpha_{2}=32^{\circ}$. The knee point yields the best compromise among the safety, design robustness, and cost. At

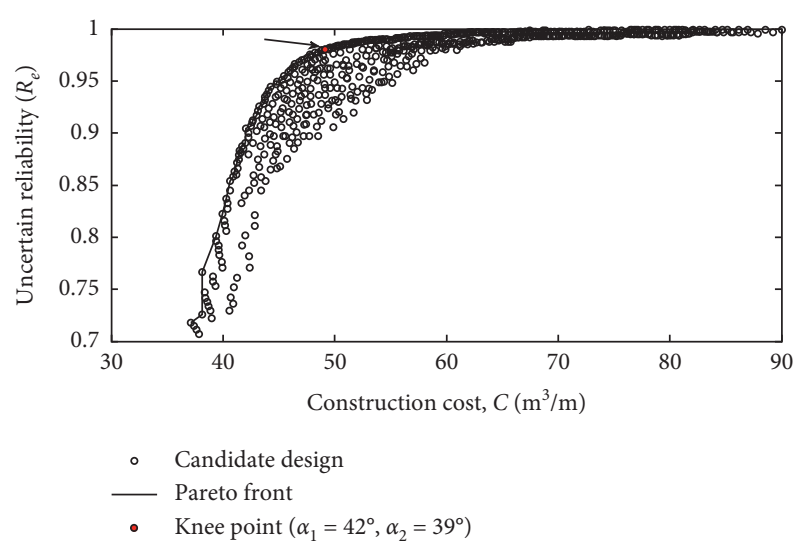

FIGURE 8: Relationship between uncertain reliability and cost of candidate designs.

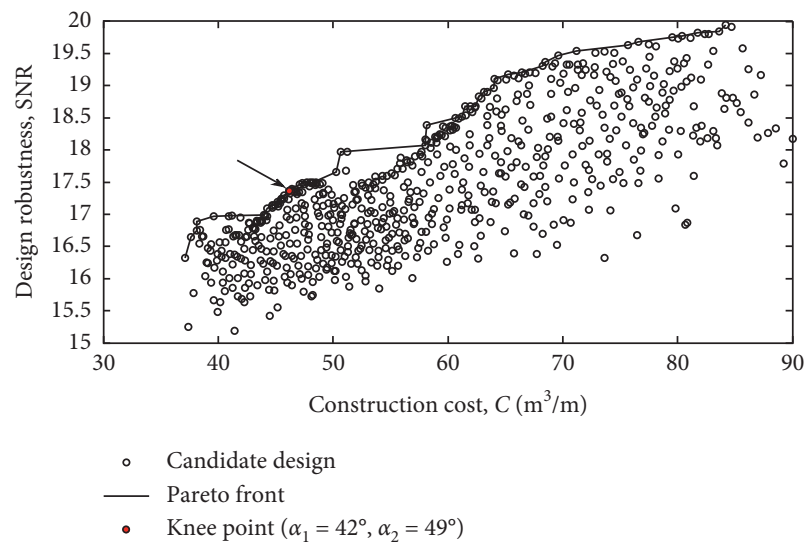

FIgURE 9: Relationship between design robustness and cost of candidate designs.

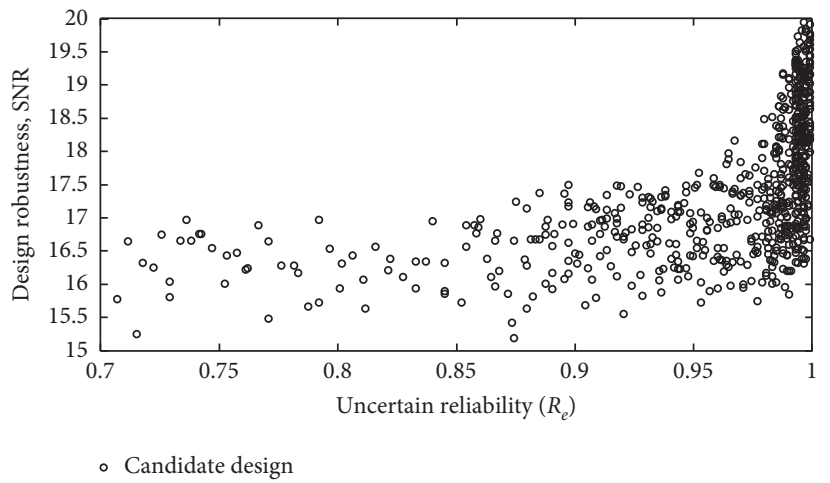

FIGURE 10: Relationship between uncertain reliability and design robustness.

this knee point, the uncertain reliability, design robustness, and the cost are $0.96,16.54$, and $46.26 \mathrm{~m}^{3} / \mathrm{m}$, respectively.

In practice, the engineers often most concern about the safety of slopes and may prefer to apply a constraint in terms of the uncertain reliability of a specific limiting value. Thus, based on this consideration, various target levels of uncertain reliability are adopted as constraints during the optimization 
TABLE 2: The results of the Pareto fronts and the knee points with the RGDS.

\begin{tabular}{|c|c|c|c|c|c|c|}
\hline \multirow{2}{*}{ Relationship } & \multirow{2}{*}{ Number of nondominated optimal designs } & \multicolumn{2}{|c|}{$\begin{array}{l}\text { Knee point on the } \\
\text { Pareto front }\end{array}$} & \multicolumn{3}{|c|}{ Objectives at the knee point } \\
\hline & & $\alpha_{1}\left({ }^{\circ}\right)$ & $\alpha_{2}\left({ }^{\circ}\right)$ & $R_{e}$ & SNR & $\mathrm{C}\left(\mathrm{m}^{3} / \mathrm{m}\right)$ \\
\hline C vs. $R_{e}$ & 64 & 42 & 39 & 0.98 & - & 49.12 \\
\hline C vs. SNR & 47 & 42 & 49 & - & 17.37 & 46.20 \\
\hline$R_{e}$ vs. SNR & $2^{*}$ & - & - & - & - & - \\
\hline
\end{tabular}

${ }^{*}$ This Pareto front includes only two designs: $R_{e}=0.997, \mathrm{SNR}=19.95$ and $R_{e}=0.999, \mathrm{SNR}=19.91$.

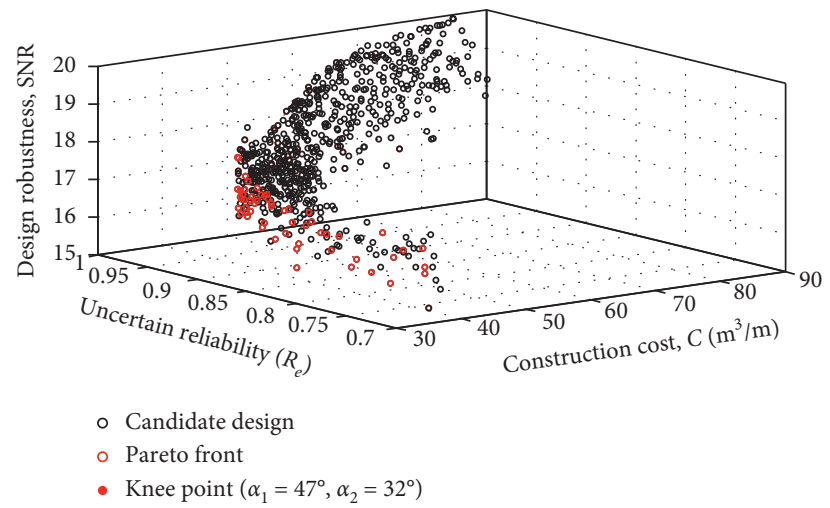

Figure 11: Three-dimensional graph for triobjective robustness optimization of soil slope design.

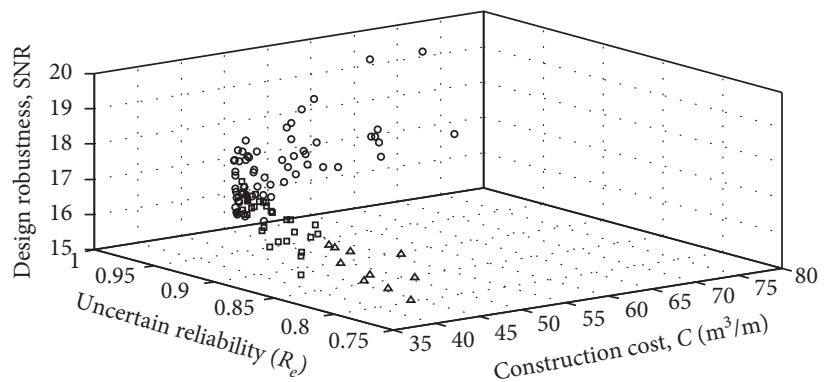

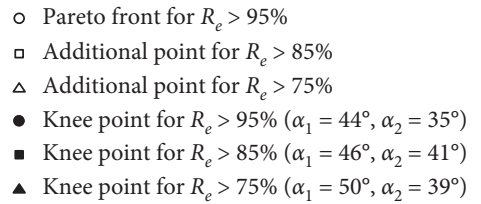

Figure 12: The Pareto fronts for different reliability constraint levels.

process. Through using various target levels, a degree of flexibility can be incorporated into the RGDS process to take into account the allowable risk of slope failure, meanwhile, to verify the reliability of the RGDS results.

For demonstration purposes, the RGDS optimization is implemented with three levels of uncertain reliability to perform various reliability constraints, i.e., $R_{e}>0.75,0.85$, 0.95. The resulting Pareto fronts and knee points under these constraints are illustrated in Figure 12, with detailed designs for different reliability constraints listed in Table 3 . It is noted that the Pareto front for the constraint of $R_{e}>0.75$ has 10 points more than that for constraint of $R_{e}>0.85$ (see the points marked by triangles in Figure 12), while the Pareto front for the constraint of $R_{e}>0.85$ has 28 points more than that for constraint of $R_{e}>0.95$ (see the points marked by squares in Figure 12). Furthermore, the knee points obtained for the Pareto fronts with the cases of $R_{e}>0.75, R_{e}>0.85$, and $R_{e}>0.95$ are, respectively, $(0.84,16.34,40.30),(0.92$, $16.64,43.74)$, and $(0.98,16.74,48.23)$, which corresponds to the design parameters $\alpha_{1}=50^{\circ}$ and $\alpha_{2}=39^{\circ}, \alpha_{1}=46^{\circ}$ and $\alpha_{2}=41^{\circ}, \alpha_{1}=44^{\circ}$ and $\alpha_{2}=35^{\circ}$, respectively.

Based on the above analysis and results, it can be found that the resulting feasible designs, Pareto fronts, and knee points are all affected by the choice of the target uncertain 
TABLe 3: The results of the Pareto fronts and the knee points with the RGDS.

\begin{tabular}{|c|c|c|c|c|c|c|c|}
\hline \multirow[t]{2}{*}{$\begin{array}{l}\text { Reliability } \\
\text { constraint }\end{array}$} & \multirow[t]{2}{*}{ Number of feasible designs } & \multirow[t]{2}{*}{ Number of nondominated optimal designs } & \multicolumn{2}{|c|}{$\begin{array}{l}\text { Knee point } \\
\text { on the } \\
\text { Pareto front }\end{array}$} & \multicolumn{3}{|c|}{ Objectives at the knee point } \\
\hline & & & $\alpha_{1}\left({ }^{\circ}\right)$ & $\alpha_{2}\left({ }^{\circ}\right)$ & Actual $R_{e}$ & SNR & $C\left(\mathrm{~m}^{3} / \mathrm{m}\right)$ \\
\hline$R_{e}>0.75$ & 662 & 99 & 50 & 39 & 0.84 & 16.34 & 40.30 \\
\hline$R_{e}>0.85$ & 631 & 89 & 46 & 41 & 0.92 & 16.64 & 43.74 \\
\hline$R_{e}>0.95$ & 489 & 61 & 44 & 35 & 0.98 & 16.74 & 48.23 \\
\hline
\end{tabular}

reliability. Moreover, as the target uncertain reliability is increased, fewer feasible designs and nondominated optimal designs could be secured, and the most-preferred design maybe has a higher cost and higher robustness. Note that the analysis results agree with those in the literature [14].

The RGDS of the soil slopes is translated into a triobjective optimization problem, with knowledge of uncertain soil parameters. In the proposed model, all three objectives, safety $\left(R_{e}\right)$, design robustness (SNR), and cost $(C)$, are to be considered and optimized. The optimization generally yields a 3D Pareto front determined by a collection of nondominated optimal designs, and the trade-off relationship between safety, design robustness, and cost can be observed and used to select the most-preferred design (knee point). In addition, the resulting most-preferred design (or knee point on the Pareto front) can be checked for the demand of not exceeding the target safety level.

\section{Conclusion}

This paper is oriented to study an RGDS approach and demonstrate its validity in the design of soil slopes. Safety, design robustness, and construction cost are all taken into account and optimized in the design of soil slopes using the RGDS approach. The findings of this research warrant the following conclusions:

(1) The proposed RGDS approach can provide an exercisable design of soil slopes, which ensures the safety, the design robust, and the cost efficiency. The validity of the RGDS approach has been demonstrated by a case of a two-layer soil slope.

(2) The RGDS of soil slopes can be attributed to a multiobjective optimization problem, and its solutions constitute a Pareto front. The knee point on the Pareto front can be further identified to yield the best-compromise design among uncertain reliability, design robustness, and excavation cost.

(3) The RGDS method requires only very small extent information about uncertain soil parameters to assess the stability of soil slopes. The approach can be implemented and processed in a deterministic manner and is computationally efficient and, therefore, makes a practical design tool.

\section{Data Availability}

The data used to support the findings of this study are available from the corresponding author upon reasonable request.

\section{Conflicts of Interest}

The authors declare that there are no conflicts of interest regarding the publication of this article.

\section{Acknowledgments}

This work was supported by the National Natural Science Foundation of China under Grant no. 11772117, Humanity and Social Science Youth Foundation of Ministry of Education of China under Grant no. 19YJC630011, and Ph.D. Foundation of Huanggang Normal University.

\section{References}

[1] C. H. Juang, J. Zhang, and W. Gong, "Reliability-based assessment of stability of slopes," IOP Conference Series: Earth and Environmental Science, vol. 26, no. 1, Article ID 012006, 2015.

[2] G. Xie, Stochastic Analysis of Geotechnical Parameters and Study on Stable Reliability of Slope, Central South University, Changsha, China, 2009.

[3] K. S. Li and P. Lumb, "Probabilistic design of slopes," Canadian Geotechnical Journal, vol. 24, no. 4, pp. 520-535, 1987.

[4] Y. Oka and T. H. Wu, "System reliability of slope stability," Journal of Geotechnical Engineering, vol. 116, no. 8, pp. 1185-1189, 1990.

[5] J. T. Christian, C. C. Ladd, and G. B. Baecher, "Reliability applied to slope stability analysis," Journal of Geotechnical Engineering, vol. 120, no. 12, pp. 2180-2207, 1994.

[6] G. B. Baecher and J. T. Christian, Reliability and Statistics in Geotechnical Engineering, Wiley, New York, NY, USA, 2003.

[7] J. T. Christian, "Geotechnical engineering reliability: how well do we know what we are doing?" Journal of Geotechnical and Geoenvironmental Engineering, vol. 130, no. 10, pp. 985-1003, 2004.

[8] A. H. S. Ang and W. H. Tang, Probability Concepts in Engineering: Emphasis on Applications to Civil and Environmental Engineering, Wiley, New York, NY, USA, 2nd edition, 2007.

[9] M. Kacewicz, "Fuzzy slope stability method," Mathematical Geology, vol. 19, no. 8, pp. 757-767, 1987.

[10] C. H. Juang, Y.-Y. Jhi, and D.-H. Lee, "Stability analysis of existing slopes considering uncertainty," Engineering Geology, vol. 49, no. 2, pp. 111-122, 1998.

[11] G. R. Dodagoudar and G. Venkatachalam, "Reliability analysis of slopes using fuzzy sets theory," Computers and Geotechnics, vol. 27, no. 2, pp. 101-115, 2000.

[12] C. I. Giasi, P. Masi, and C. Cherubini, "Probabilistic and fuzzy reliability analysis of a sample slope near Aliano," Engineering Geology, vol. 67, no. 3-4, pp. 391-402, 2003. 
[13] H. J. Park, J.-G. Um, I. Woo, and J. W. Kim, "Application of fuzzy set theory to evaluate the probability of failure in rock slopes," Engineering Geology, vol. 125, pp. 92-101, 2012.

[14] W. Gong, L. Wang, S. Khoshnevisan, C. H. Juang, H. Huang, and J. Zhang, "Robust geotechnical design of earth slopes using fuzzy sets," Journal of Geotechnical and Geoenvironmental Engineering, vol. 141, no. 1, Article ID 04014084, 2014.

[15] N. O. Nawari and R. Liang, "Fuzzy-based approach for determination of characteristic values of measured geotechnical parameters," Canadian Geotechnical Journal, vol. 37, no. 5, pp. 1131-1140, 2000.

[16] B. Liu, Uncertainty Theory, Springer, Berlin, Germany, 2nd edition, 2007.

[17] B. Liu, Uncertainty Theory, Springer, Berlin, Germany, 4th edition, 2015.

[18] Z. Liu, R. Zhao, X. Liu, and L. Chen, "Contract designing for a supply chain with uncertain information based on confidence level," Applied Soft Computing, vol. 56, pp. 617-631, 2017.

[19] X. Wu, R. Zhao, and W. Tang, "Optimal contracts for the agency problem with multiple uncertain information," Knowledge-Based Systems, vol. 59, no. 2, pp. 161-172, 2014.

[20] K. Yang, R. Zhao, and Y. Lan, "The impact of risk attitude in new product development under dual information asymmetry," Computers \& Industrial Engineering, vol. 76, no. 3, pp. 122-137, 2014.

[21] X. Zhou, W. Huang, Z. Liu, and H. Chen, "Assessment of slope stability under uncertain circumstances," Soft Computing, vol. 22, no. 17, pp. 5735-5745, 2018.

[22] X. Zhou, J. Li, Z. Liu, and Y. Tang, "Analysis of slope stability with imprecise soil properties using uncertain sets," Mathematical Problems in Engineering, vol. 2019, pp. 1-9, 2019.

[23] C. H. Juang, L. Wang, S. Khoshnevisan, and S. Atamturktur, "Robust geotechnical design Methodology and applications," Journal of Geotechnical Engineering, vol. 8, no. 3, pp. 71-81, 2013.

[24] H. G. Beyer and B. Sendhoff, "Robust optimization-a comprehensive survey," Computer Methods in Applied Mechanics and Engineering, vol. 196, no. 33-34, pp. 3190-3218, 2007.

[25] G. Taguchi, Introduction to Quality Engineering: Designing Quality into Products and Processes, Quality Resources, White Plains, NY, 1986.

[26] K.-L. Tsui, "An overview of Taguchi method and newly developed statistical methods for robust design," IIE Transactions, vol. 24, no. 5, pp. 44-57, 1992.

[27] A. W. Bishop, "The use of the slip circle in the stability analysis of slopes," Géotechnique, vol. 5, no. 1, pp. 7-17, 1955.

[28] W. Fellenius, "Calculation of the stability of earth slope," in Proceedings of the Transactions of 2nd Congress on Large Dams, pp. 445-462, Washington, DC, 1936.

[29] N. Janbu, "Application of composite slip surface for stability analysis," in Proceedings of the European Conference on Stability of Earth Slopes, pp. 43-49, Stockholm, Sweden, 1954.

[30] E. Spencer, "A method of analysis of the stability of embankments assuming parallel inter-slice forces," Géotechnique, vol. 17, no. 1, pp. 11-26, 1967.

[31] Y. H. Huang, Stability Analysis of Earth Slopes, Springer US, Boston, MA, USA, 1983.

[32] H. El-Ramly, N. R. Morgenstern, and D. M. Cruden, "Probabilistic assessment of stability of a cut slope in residual soil," Géotechnique, vol. 55, no. 1, pp. 77-84, 2005.

[33] GEO-SLOPE International Ltd., Stability Modeling with SLOPE/W: An Engineering Methodology, GEO-SLOPE International Ltd., Calgary, Canada, 2012.
[34] Y. J. Li, M. A. Hicks, and J. D. Nuttall, "Comparative analyses of slope reliability in 3D," Engineering Geology, vol. 196, pp. 12-23, 2015.

[35] M. A. Hicks and Y. Li, "Influence of length effect on embankment slope reliability in 3D," International Journal for Numerical and Analytical Methods in Geomechanics, vol. 42, no. 7, pp. 891-915, 2018.

[36] A. Ghosh and S. Dehuri, "Evolutionary algorithms for multicriterion optimization: a survey," International Journal of Computing and Information Sciences, vol. 2, no. 1, pp. 38-57, 2004.

[37] F. Y. Cheng and D. Li, "Multiobjective optimization design with Pareto genetic algorithm," Journal of Structural Engineering, vol. 123, no. 9, pp. 1252-1261, 1997.

[38] K. Deb, A. Pratap, S. Agarwal, and T. Meyarivan, "A fast and elitist multiobjective genetic algorithm: NSGA-II," IEEE Transactions on Evolutionary Computation, vol. 6, no. 2, pp. 182-197, 2002.

[39] P. L. Yu, "A class of solutions for group decision problems," Management Science, vol. 19, no. 8, pp. 936-946, 1973.

[40] K. Miettinen, Nonlinear Multiobjective Optimization, Kluwer, Boston, MA, USA, 1999.

[41] K. Deb, Multi-objective Optimization Using Evolutionary Algorithms, Wiley, Chichester, UK, 2001.

[42] J. Branke, K. Deb, H. Dierolf, and M. Osswald, "Finding knees in multi-objective optimization," in Proceedings of the Parallel Problem Solving from Nature VIII, Springer, Berlin, Germany, pp. 722-731, 2004.

[43] Y. J. Li, M. A. Hicks, and P. J. Vardon, "Uncertainty reduction and sampling efficiency in slope designs using 3D conditional random fields," Computers and Geotechnics, vol. 79, pp. 159-172, 2016. 